\title{
South American
}

National Cancer Institute

\section{Source}

National Cancer Institute. South American. NCI Thesaurus. Code C126532.

Denotes a person whose ancestry is in any of the countries of South America. 